\title{
On Fixed-Price Marketing for Goods with Positive Network Externalities.
}

\author{
Vahab S. Mirrokni, Sebastien Roch^, Mukund Sundararajan \\ ${ }^{1}$ Google Research, New York \\ 2 Department of Mathematics, UW-Madison \\ ${ }^{3}$ Google Research, Mountain View
}

\begin{abstract}
In this paper we discuss marketing strategies for goods that have positive network externalities, i.e., when a buyer's value for an item is positively influenced by others owning the item. We investigate revenue-optimal strategies of a specific form where the seller gives the item for free to a set of users, and then sets a fixed price for the rest. We present a $\frac{1}{2}$-approximation for this problem under assumptions about the form of the externality. To do so, we apply ideas from the influence maximization literature [13] and also use a recent result on non-negative submodular maximization as a blax-box $[3,7]$.
\end{abstract}

\section{Introduction}

Consumer goods and services often exhibit positive network externalities - a buyer's value for the good or service is influenced positively by other buyers owning the good or using the service. Such positive network externalities arise in various ways. For instance, XBox Live is an online gaming service that allows users to play with each other. Thus, the value of an XBox to a user increases as more of her friends also own an XBox. Popular smartphone platforms (such as Android, iOS, or Windows Mobile) actively support developer networks, because developers add 'Applications' that make the phone more useful to other users. Thus, the value of a smartphone to a user increases with the size of the developer network. Many consumer goods, especially those that have been newly introduced, benefit from word-of-mouth effects. Prospective buyers use this word-of-mouth to judge the quality of the item while making a purchase decision. If the good or service is of good quality, the word-of-mouth will cause a positive externality.

Irrespective of how positive network externalities arise, it is clear that they are worth paying attention to in designing a good marketing/pricing strategy. Companies that own smartphone platforms often hand out upcoming devices to developers. Manufacturers send out a new version of a device to technology review websites. Detergent companies, and manufacturers of health foods, hand out free samples of new products. The hope is that giving out the item for free

\footnotetext{
* Work supported by NSF grant DMS-1007144 and an Alfred P. Sloan Research Fellowship. Part of this work was done at Microsoft Research.
} 
drives up demand for the good/service and increases the revenue generated from future sales.

In this paper we attempt to identify a revenue maximizing marketing strategy of the following form: The seller selects a set $S$ of buyers and gives them the good for free, and then sets a fixed per-unit price $p$ at which other consumers can buy the item. The strategy is consistent with practice as the examples above illustrate and is easy to implement. However, optimizing revenue poses two challenges. First, the choice of the set $S$ and the price $p$ are coupled and must be traded-off optimally: expanding the set $S$ loses potential revenue from the set $S$, but may increase the positive externality on buyers not in $S$ and may allow the seller to extract more revenue from them. A second, more subtle, issue is that it is important to have a handle on the dynamics of adoption. For a fixed set $S$ and a price $p$, a buyer $j \notin S$ who is initially unwilling to buy the item at a price $p$, may later do so as other buyers (who are not in $S$ and are willing to buy the item at a price $p$ ) go first. This may result in a 'cascade' of sales and it is important to have a handle on this revenue when optimizing for $S$ and $p$.

Our Results. The related problem of influence maximization (as opposed to our revenue maximization problem) is well-studied (e.g., Chapter 23 in [13]). The canonical question in this literature, first posed by Domingos and Richardson [5], is: Which set $I$ of influential nodes of cardinality $k$ in a social network should be convinced to use a service, so that subsequent adoption of the service is maximized? This literature has made substantial progress in understanding the cascading of process of adoption and using this to optimize for $I$ (see for instance $[5,11,12,15])$. However, this literature does not model the impact of price on the probabiity of adopting a service and does not attempt to quantify the revenue from adoption. Therefore it cannot be directly applied to answer our revenue-maximization question.

Our main technical contribution (Lemma 1) establishes a correspondence between the dynamics of our (price-sensitive) process and the dynamics of the general threshold model [11] from the influence maximization literature. We use it along with a recent result on optimizing non-negative submodular functions $[3,7]$ to identify an algorithm that is a $\frac{1}{2}$-approximation for our problem (Theorem 1). It is worth noting that, although we prove our result through establishing a connection to the general threshold model [11], we cannot use the greedy $\left(1-\frac{1}{e}\right)$-approximation algorithm of Nemhauser, Wolsey, and Fischer [16], and instead we need to use the recent $\frac{1}{2}$-approximation [3,7] for non-negative submodular maximization.

More Related Work. Besides the literature on influence maximization mentioned above, there is also an expanding literature on algorithms for revenue maximization with positive network externalities. Hartline, Mirrokni, and Sundararajan [9] study the marketing strategies where the seller can give the item for free to a set of buyers, and then visit the remaining buyers in a sequence offering each a buyer-specific price. Such strategies are hard to implement because the seller must control the time at which the transaction takes place. Further, there is also evidence that buyers may react negatively to price-discrimination as 
it generates a perception of unfairness. Oliver and Shor [17] discuss why such a negative reaction may arise. Partly in response to some of these issues, Akhlaghpour et al. [1] explore strategies that allow the seller to vary the price across time. Though these strategies do not perform price discrimination, there is some evidence that such strategies may also cause buyers to react negatively, especially if the prices vary significantly across time. For instance, there was some unhappiness when Apple dropped the price of an iPhone by $33 \%$ two months after an initial launch (http://www.apple.com/hotnews/openiphoneletter/). In contrast, our approach is to offer the good at a fixed price, albeit after giving the item for free to some set of users, a step which seems socially acceptable (see the examples in the Introduction.) This strategy can also increase the revenue to the seller above using a fixed price without an influence step (see Appendix A for an example illustrating this). More recently, Haghpanah et al. [8] take an auction-theoretic (as opposed to a pricing) approach. This approach is applied only to some forms of positive externality where the temporal sequence of sales is not necessary for the externality to manifest (so it applies to the XBox example from the introduction, but not the settings where word-of-mouth is involved).

There is also a literature in economics that has studied equilibrium behavior in the adoption of goods with network externalities $[2,4,6,10,14,18]$. For instance, Carbal, Salant, and Woroch [4] show that in a social network the seller might decide to start with low introductory prices to attract a critical mass of players when the players are large (i.e, the network effect is significant). The focus here is to characterize the equilibrium that arises from buyer rationality, as opposed to optimizing the seller's strategy.

\section{Model}

Consider a seller who wants to sell a good to a set of potential buyers, $V$. Consider a digital good with zero marginal cost of manufacturing and assume that the seller has an unlimited supply of the good. We assume that the seller is a monopolist and is interested in maximizing its revenue.

\subsection{Externality Model}

We assume that a buyer $i$ 's value for the digital good depends on its own inherent valuation $\omega_{i}$ for the good and also on the influence from the set $S \subseteq V \backslash\{i\}$ of buyers who already own the good. More specifically, we consider the graph model with concave influence in which each buyer $i \in V$ is associated with a non-negative, non-decreasing, concave function $f_{i}: \mathbb{R}_{+} \rightarrow \mathbb{R}_{+}$with $f_{i}(0)=0$. The value of the digital item for a buyer $i \in V$ given that a set $S$ of buyers have already bought the item is denoted by $v_{i}(S)$ and is equal to $\omega_{i}+f_{i}\left(\sum_{j \in S} w_{i j}\right)$. Each inherent valuation $\omega_{i}$ is drawn independently from a uniform distribution (or more generally from a distribution $G_{i}$ ) and each $w_{i j}$ is drawn from a distribution $\widetilde{G}_{i j}$ capturing the influence of buyer $i$ over buyer $j$. We assume that a buyer $i$ buys the item at a price $p$ if and only if $v_{i}(S) \geq p$. We assume that the 
valuations and prices are in an interval $[0, M]$. Throughout this paper, we fix $M=1$ for convenience.

\section{$2.2 \quad$ Fixed-Price Marketing}

A fixed-price marketing strategy consists of two stages:

Inital Influence. In this stage, the seller gives the item for free to a subset $A$ of buyers.

Price Setting. In this stage, the seller sets a fixed price $p$ for the digital good.

After setting the price $p$, buyers $i$ with value $v_{i}(A) \geq p$ buy the item. Let set $S_{1}$ be the set of buyers whose value $v_{i}(A)$ after the influence step is greater than $p$, i.e., $S_{1}=\left\{i \notin A \mid v_{i}(A) \geq p\right\}$. After buyers in set $S_{1}$ buying the item at price $p$, they may influence other buyers, and their value may increase and go above $p$. As a result, after set $S_{1}$ buys the item, some other buyers may have incentive to buy the item. Let set $S_{2}$ be this set of buyers, i.e., $S_{2}=\left\{i \notin A \cup S_{1} \mid v_{i}\left(A \cup S_{1}\right) \geq p\right\}$. As more buyers buy the digital good, more buyers have incentive to buy the item. This process continues and the dynamics propagates, i.e, for any $i(2 \leq i \leq k)$, $S_{i}$ is the set of buyers not in $\left(\cup_{j<i} S_{j}\right) \cup A$ whose value is more than or equal to $p$ given that set $\left(\cup_{j<i} S_{j}\right) \cup A$ of buyers already adopted the item. The seller's goal is to find a set $A$ of buyers to influence and a fixed price $p$ to maximize the total revenue he can extract from buyers, i.e., in the optimal fixed-price marketing problem with positive network externalities, the sellers's goal is to choose $A$ and $p$ to maximize $p\left(\left|\cup_{i \geq 1} S_{i}\right|\right)$.

\section{Approximation Algorithm}

In this section, we design a constant-factor approximation algorithm for the problem. We first observe that a simple $\frac{1}{8}$-approximation algorithm exists for the special case of the problem where weights are deterministic. Then we elaborate on an improved $\frac{1}{2}$-approximation algorithm for the graph model with concave influence function that explicitly exploits dynamics.

Sketch of a simple $\frac{1}{8}$-approximation algorithm. For fixed $\omega_{i}$ 's and $w_{i j}$ 's, a randomized $\frac{1}{8}$-approximation algorithm is easily derived: Give the item for free to each buyer with probability $1 / 2$ independently, then search for the highest revenue achievable given the freebies by considering all prices over a $1 / \operatorname{poly}(n)$ grid. Let $A_{*}, p_{*}$ be an optimal solution to the problem and define $B_{*}=\{i \in$ $\left.V: \omega_{i}+f_{i}\left(\sum_{j} w_{i j}\right) \geq p_{*}\right\}$. In expectation, there are $\left|B_{*}\right| / 2$ remaining potential buyers after the first stage. We claim that, for a fixed second-stage price of $p_{*} / 2$, each of the remaining nodes in $B_{*}$ has a probability $\frac{1}{2}$ of reaching value $p_{*} / 2$ in the second stage giving an expected revenue of $\left|B_{*}\right| p_{*} / 8$ and proving the claim. Indeed, let $\mathcal{P}_{i}$ be the revenue earned from $i$ when $p=p_{*} / 2$ and note that, ignoring dynamics (i.e., considering only the first round following the influence stage),

$$
\mathbb{E}\left[\mathcal{P}_{i}\right] \geq \frac{p_{*}}{4} \mathbb{P}\left[\omega_{i}+f_{i}\left(\sum_{j} \mathbb{1}_{j} w_{i j}\right) \geq \frac{p_{*}}{2}\right]
$$


where $\mathbb{1}_{i}$ is 1 if $i$ gets the item for free, and 0 otherwise (and $w_{i i}=0$ ). Noting that

$$
\sum_{j} \mathbb{1}_{j} w_{i j} \geq \frac{1}{2} \sum_{j} w_{i j} \Longrightarrow f_{i}\left(\sum_{j} \mathbb{1}_{j} w_{i j}\right) \geq \frac{1}{2} f_{i}\left(\sum_{j} w_{i j}\right) \geq \frac{1}{2}\left[p_{*}-\omega_{i}\right],
$$

where we used the concavity of $f_{i}$ and the definition of $B_{*}$, we get

$$
\mathbb{P}\left[\omega_{i}+f_{i}\left(\sum_{j} \mathbb{1}_{j} w_{i j}\right) \geq \frac{p_{*}}{2}\right] \geq \mathbb{P}\left[\sum_{j} \mathbb{1}_{j} w_{i j} \geq \frac{1}{2} \sum_{j} w_{i j}\right] \geq 1 / 2,
$$

by symmetry.

A $\frac{1}{2}$-approximation algorithm. Now we present an improved $\frac{1}{2}$-approximation algorithm when the weights are random that explicitly exploits the dynamics of the influence process, unlike the simple algorithm above. We assume further that the prices are in an interval $[0, M]$ for some constant $M$, that the $w_{i j}$ 's are drawn from arbitrary distributions and that the $\omega_{i}$ 's are drawn from a uniform distribution over $[0, M]$. For convenience, we take $M=1$. For any price $p \in[0,1]$, consider the following set function $Y_{p}: 2^{V} \rightarrow \mathbb{R}_{+}$: for any subset $A \subset V, Y_{p}(A)$ is the expected revenue from giving the item for free to set $A$ in the influence stage, and setting the price to $p$ in the fixed-price stage. Our algorithm is as follows. Fix $\epsilon=o\left(n^{-1}\right)$.

1. For every integer $\rho$ where $0 \leq \rho \leq \epsilon^{-1}$ do:

- Given that the price in the second stage is $p=\rho \epsilon$, using the approximation algorithm for non-negative submodular maximization in [3,7], find a set $A_{\rho}$ of users to influence in the first stage. The algorithm in [3, 7] uses oracle calls to the objective function. We simulate oracle calls to $Y_{p}$ by running the influence process $\operatorname{poly}(n)$ times independently and averaging.

- Let $L_{\rho}$ be the revenue from giving the item to set $A_{\rho}$ and setting price $p=\rho \epsilon$.

2. Output the set $A_{\rho}$ and price $\rho \epsilon$ for which $L_{\rho}$ is maximized.

Our approximation result follows from a mapping of the fixed-price strategy to a model of viral marketing introduced in $[11,12]$. In the viral marketing problem, one gives an item for free to a group of individuals as we do here but, in the subsequent influence stage, revenue is ignored (i.e., there is no price) and instead one aims to maximize the number of individuals who purchase the product. In $[11,12]$, the general threshold model was introduced to model the influence process. Formally, the special case of the general threshold model relevant here is obtained from our influence process by setting $p=0$ and letting $\omega_{i}$ be uniform in $[-1,0]$. See $[11,12]$ for more details on the general threshold model.

Theorem 1 (Approximation). The above algorithm is a $\frac{1}{2}$-approximation algorithm for the optimal fixed-price marketing problem with positive network externalities in the graph model with concave influence.

It is worth noting that, although we prove our result through establishing a connection to the general threshold model, the final set function that we need 
to maximize is not necessarily monotone. Therefore, unlike the viral marketing problem in $[11,12]$, we cannot use the greedy $\left(1-\frac{1}{e}\right)$-approximation algorithm of Nemhauser, Wolsey, and Fischer [16] for monotone submodular maximization subject to cardinality constraints. Instead we use the local search $\frac{1}{2}$ approximation $[3,7]$ for non-negative submodular maximization. Before stating the proof of this theorem, we note that the approximation algorithm applies to a more general setting for the distribution of inherent valuations $\omega_{i}$ 's.

Remark 1. Our $\frac{1}{2}$-approximation algorithm holds more generally under the assumption that the inherent valuations $\omega_{i}$ are random with distribution $G_{i}$ with positive, differentiable, non-decreasing density $g_{i}$ on $(0,1)$ and, further, that there is a constant $\bar{g}>0$ such that the $g_{i}$ 's are bounded above by $\bar{g}$. Our proof is given under these assumptions. The obvious open question is to see if the assumption that $g_{i}$ is non-decreasing can be relaxed to a more realistic assumption like the monotone hazard rate condition.

Proof. Note that it follows from Chebyshev's inequality and the fact that the revenue is bounded by $n$ that our simulated oracle calls are accurate within $1 / \operatorname{poly}(n)$ with probability $1-1 / \operatorname{poly}(n)$. Let OPT be the optimal revenue. We first condition on the edge weights $\left\{w_{i j}\right\}_{i j}$.

Proposition 1 (Submodularity of $Y_{p}$ ). Conditioned on the edge weights $\left\{w_{i j}\right\}_{i j}$, the function $Y_{p}$ is a (not necessarily monotone) non-negative, submodular function.

Proposition 2 (Continuity of $Y_{p}$ ). Let $\delta_{n}$ be a vanishing function of $n$ (possibly negative) with $\left|\delta_{n}\right|=o\left(n^{-k}\right)$ with $k \geq 1$. Conditioned on the edge weights $\left\{w_{i j}\right\}_{i j}$, we have

$$
\left|Y_{p}(S)-Y_{p+\delta_{n}}(S)\right|=o\left(n^{-k}\right) \mathrm{OPT},
$$

for any set $S$ of buyers.

By linearity, both propositions still hold after taking expectation over edge weights. Theorem 1 then follows from the main result in $[3,7]$ where a $\frac{1}{2}$ approximation algorithm is derived for non-negative submodular maximization.

It remains to prove the propositions.

Proof. (of Proposition 1) For any price $p$ and any buyer $i$, consider the following set function $h_{p}^{i}: 2^{V \backslash\{i\}} \rightarrow \mathbb{R}_{+}$: for any subset $A \subset V \backslash\{i\}, h_{p}^{i}(A)$ is the expected revenue from user $i$ if we give the item for free to set $A$ in the influence stage, and then set the price $p$ in the second stage. For any set $A$,

$$
Y_{p}(A)=\sum_{i \in V \backslash A} h_{p}^{i}(A)
$$

We need the following lemma

Lemma 1. The set functions $h_{p}^{i}$ for any buyer $i$ are monotone and submodular. 
Proof. Fix $0 \leq p \leq 1$. Let $S$ be a set of buyers. Note that

$$
\omega_{i}+f_{i}\left(\sum_{j \in S} w_{i j}\right) \geq p,
$$

if and only if

$$
f_{i}\left(\sum_{j \in S} w_{i j}\right) \geq \max \left\{0, p-\omega_{i}\right\} \equiv \omega_{i, p} .
$$

Denote by $Q_{i, p}$ the distribution function of $\omega_{i, p}$. Note that

$$
Q_{i, p}(x)=1-G_{i}(p-x),
$$

for $0 \leq x<p$ and $Q_{i, p}(x)=1$ for $x \geq p$. By assumption, on $(0, p), Q_{i, p}^{\prime}(x)=$ $g_{i}(p-x)>0$ and $Q_{i, p}^{\prime \prime}(x)=-g_{i}^{\prime}(p-x) \leq 0$ so that $Q_{i, p}$ is increasing and concave. Further, since $Q_{i, p}$ is continuous at $p$ and constant for $x \geq p, Q_{i, p}$ is non-decreasing and concave on $[0,+\infty)$.

Let $U_{i}, i \in V$, be independent uniform random variables. We now describe a mapping of our influence process to a special case of the general threshold model where a user $i$ adopts a product as soon as $Z_{i}\left(\sum_{j \in S} w_{i j}\right) \geq U_{i}$ for a concave function $Z_{i}$. To transfer the randomness of our inherent valuation to the threshold side of the general threshold model, we use the inverse transform method where one simulates a random variable $X$ with distribution function $H$ by using $H^{-1}(U)$ where $U$ is uniform in $[0,1]$ and $H^{-1}$ is a generalized inverse function. By definition of $Q_{i, p}$,

$$
\begin{aligned}
\mathbb{P}\left[Q_{i, p}\left(f_{i}\left(\sum_{j \in S} w_{i j}\right)\right) \geq U_{i}\right] & =\mathbb{P}\left[f_{i}\left(\sum_{j \in S} w_{i j}\right) \geq \omega_{i, p}\right] \\
& =\mathbb{P}\left[\omega_{i}+f_{i}\left(\sum_{j \in S} w_{i j}\right) \geq p\right] .
\end{aligned}
$$

Since $Q_{i, p}$ and $f_{i}$ are non-decreasing and concave, the composition $Q_{i, p}\left(f_{i}(\cdot)\right)$ is concave as well and $Q_{i, p}\left(f_{i}\left(\sum_{j \in S} w_{i j}\right)\right)$ is submodular in $S$. Hence, we have shown that for any fixed $p$, the dynamics of the influence stage are equivalent to a submodular general threshold model. In particular, by the results in [15], we have that $h_{p}^{i}$ is submodular.

We finish the proof of Proposition 1 by proving the following lemma.

Lemma 2. If all set functions $h_{p}^{i}$ for $i \in V$ are monotone and sub modular, then the set function $Y_{p}$ is also sub modular (but not monotone).

The proof, which is similar to the proof of a similar lemma in [9], can be found in the appendix.

The proof of Proposition 2 can be found in the appendix. 


\section{References}

1. Hessameddin Akhlaghpour, Mohammad Ghodsi, Nima Haghpanah, Vahab S. Mirrokni, Hamid Mahini, and Afshin Nikzad. Optimal iterative pricing over social networks (extended abstract). In WINE, pages 415-423, 2010.

2. Bernard Bensaid and Jean-Philippe Lesne. Dynamic monopoly pricing with network externalities. International Journal of Industrial Organization, 14(6):837855, October 1996.

3. Niv Buchbinder, Moran Feldman, Joseph (Seffi) Naor, and Roy Schwartz. A tight linear time (1/2)-approximation for unconstrained submodular maximization. In FOCS '12, 2012.

4. Luis Cabral, David Salant, and Glenn Woroch. Monopoly pricing with network externalities. Industrial Organization 9411003, EconWPA, November 1994.

5. Pedro Domingos and Matt Richardson. Mining the network value of customers. In $K D D$ '01, pages 57-66, New York, NY, USA, 2001. ACM.

6. Joseph Farrell and Garth Saloner. Standardization, compatibility, and innovation. RAND Journal of Economics, 16(1):70-83, Spring 1985.

7. Uriel Feige, Vahab S. Mirrokni, and Jan Vondrak. Maximizing non-monotone submodular functions. In FOCS 'O\%, pages 461-471, 2007.

8. Nima Haghpanah, Nicole Immorlica, Vahab S. Mirrokni, and Kamesh Munagala. Optimal auctions with positive network externalities. In ACM Conference on Electronic Commerce, pages 11-20, 2011.

9. Jason D. Hartline, Vahab S. Mirrokni, and Mukund Sundararajan. Optimal marketing strategies over social networks. In $W W W$, pages 189-198, 2008.

10. Michael L Katz and Carl Shapiro. Network externalities, competition, and compatibility. American Economic Review, 75(3):424-40, June 1985.

11. David Kempe, Jon Kleinberg, and Éva Tardos. Maximizing the spread of influence through a social network. In KDD '03, pages 137-146, New York, NY, USA, 2003. ACM.

12. David Kempe, Jon Kleinberg, and Éva Tardos. Influential nodes in a diusion model for social networks. In in ICALP, pages 1127-1138. Springer Verlag, 2005.

13. J. Kleinberg. Cascading behavior in networks: algorithmic and economic issues. Cambridge University Press, 2007.

14. Robin Mason. Network externalities and the coase conjecture. European Economic Review, 44(10):1981-1992, December 2000.

15. Elchanan Mossel and Sebastien Roch. On the submodularity of influence in social networks. In STOC 'O7, pages 128-134, New York, NY, USA, 2007. ACM.

16. G. Nemhauser, L. Wolsey, and M. Fisher. An analysis of the approximations for maximizing submodular set functions. Mathematical Programming, 14:265-294, 1978.

17. Richard L. Oliver and Mikhael Shor. Digital redemption of coupons: Satisfying and dissatisfying eects of promotion codes. Journal of Product and Brand Management, 12:121-134, 2003.

18. Arun Sundararajan. Local network effects and network structure. Working paper, 2004.

\section{A Example}

The following example shows that, by giving the item for free to a subset of buyers first and then setting a public price, the seller may gain significantly more 
revenue compared to only setting a public price. Let the inherent valuations $\omega_{i}$ be drawn uniformly from $[0,1]$ and let pairwise influences $w_{i j}$ all be equal to $\frac{4 c}{n}$ (deterministically). If we only apply pricing without the initial influence step, the maximum price that the seller can set is 1 , and therefore the maximum revenue the seller can gain is $n$. However with an initial influence stage, the seller can give the item for free to half of the buyers and set the price of $2(c+1)$ which results in a revenue of $(c+1) n$ and a gap of $c+1$ between the pricing with or without the influence stage. The gap in this example can easily be boosted by setting $w_{i j}=1$ which results in a gap of $n / 4$.

\section{B Additional proofs}

Proof. (of Lemma 2) Using $Y_{p}(A)=\sum_{i \in V \backslash A} h_{p}^{i}(A)$. We need to prove that, for any set $A \subseteq V$ and $C \subseteq V$,

$$
Y_{p}(A)+Y_{p}(C) \geq Y_{p}(A \cup C)+Y_{p}(A \cap C) .
$$

First, using monotonicity of $h_{p}^{i}$, for each $i \in(A \backslash C) \cup(C \backslash A)$

$$
\sum_{i \in A \backslash C} h_{p}^{i}(C)+\sum_{i \in C \backslash A} h_{p}^{i}(A) \geq \sum_{i \in A \backslash C} h_{p}^{i}(A \cap C)+\sum_{i \in C \backslash A} h_{p}^{i}(A \cap C)
$$

Now, using the submodularity of $h_{p}^{i}$, for each $i \in V \backslash(A \cup C)$,

$$
h_{p}^{i}(A)+h_{p}^{i}(C) \geq h_{p}^{i}(A \cup C)+h_{p}^{i}(A \cap C) .
$$

Therefore, summing the above inequality for all $i \in V \backslash(A \cup C)$, we get:

$$
\begin{aligned}
& \sum_{i \in V \backslash(A \cup C)} h_{p}^{i}(A)+\sum_{i \in V \backslash(A \cup C)} h_{p}^{i}(C) \\
& \geq \sum_{i \in V \backslash(A \cup C)} h_{p}^{i}(A \cup C)+\sum_{i \in V \backslash(A \cup C)} h_{p}^{i}(A \cap C)
\end{aligned}
$$

Summing (1) and (2), we get

$$
\begin{aligned}
& \sum_{i \in V \backslash A} h_{p}^{i}(A)+\sum_{i \in V \backslash C} h_{p}^{i}(C) \\
& \geq \sum_{i \in V \backslash(A \cup C)} h_{p}^{i}(A \cup C)+\sum_{i \in V \backslash(A \cap C)} h_{p}^{i}(A \cap C) .
\end{aligned}
$$

This proves the lemma.

Proof. (of Proposition 2) Assume $\delta_{n}$ is positive. A similar argument for $\delta_{n}$ negative. For all $i$, let $\gamma_{i, p}$ be the density function of $\omega_{i, p}$ on $(0, p)$ (see the proof of Lemma 1) and let $\Gamma_{i, p}=\mathbb{P}\left[\omega_{i, p}=0\right]$. By the definition of $\omega_{i, p}$ and the monotonicity of $g, \Gamma_{i, p}$ is non-increasing in $p$ and, for each $x \in(0, p), \gamma_{i, p}(x)$ 
is non-decreasing in $p$. Hence, by a standard argument, we can couple copies of $\left\{\omega_{i, p}\right\}_{i}$ and $\left\{\omega_{i, p+\delta_{n}}\right\}_{i}$ such that all pairs $\omega_{i, p}, \omega_{i, p+\delta_{n}}$ are equal except with probability at most

$$
\begin{aligned}
\sum_{i}\left[1-\left(\Gamma_{i, p+\delta_{n}}+\int_{0}^{p} \gamma_{i, p}(x) d x\right)\right] & =\sum_{i}\left[\Gamma_{i, p}-\Gamma_{i, p+\delta_{n}}\right] \\
& =\sum_{i}\left[\int_{p}^{p+\delta_{n}} g_{i}(x) d x\right] \\
& \leq n \bar{g} \delta_{n} \\
& =o\left(n^{-k+1}\right) .
\end{aligned}
$$

In case there is a disagreement for at least one such pair, the difference in revenue is at most $p n \leq n$ and therefore the overall expected revenue satisfies

$$
\left|Y_{p}(S)-Y_{p+\delta_{n}}(S)\right|=o\left(n^{-k}\right) \mathrm{OPT},
$$

for any $S$, where we used the fact that under our assumptions OPT $=\Omega(n)$ (take $S=\emptyset$ and choose a small enough price $p$ as a function of $\bar{g}$ ). 